\title{
TITLE:
}

\section{The endoplasmic reticulum stress signaling pathways in plants.}

\author{
$\operatorname{AUTHOR}(\mathrm{S})$ : \\ Urade, Reiko
}

\section{CITATION:}

Urade, Reiko. The endoplasmic reticulum stress signaling pathways in plants.. BioFactors 2009, 35(4): 326-331

\section{ISSUE DATE:}

2009-05-04

URL:

http://hdl.handle.net/2433/89514

\section{RIGHT:}

c 2009 International Union of Biochemistry and Molecular Biology, Inc. 許諾条件により本 文は2010-05-05に公開.; This is not the published version. Please cite only the published version.; この論文は出版社版でありません。引用の際には出版社版をご確認ご利用くだ さい。 


\section{The endoplasmic reticulum stress signaling pathway in plants}

Reiko Urade

Division of Food Science and Biotechnology, Graduate School of Agriculture, Kyoto University

Gokasho, Uji, Kyoto 611-0011

Japan

Tel: +81774383757

Fax: +81774383858

E-mail: urade@kais.kyoto-u.ac.jp

Running title: ER stress signaling pathway in plants

Keywords: unfolded protein response; endoplasmic reticulum; stress signaling pathway; basic leucine zipper transcription factor 
Abstract

The unfolded protein response (UPR) is an evolutionarily conserved mechanism by which all eukaryotic cells preserve the homeostasis of the endoplasmic reticulum (ER) in the face of accumulation of unfolded proteins in the ER. Plants possess at least two signaling pathways specific for UPR. ER membrane-bound ER stress sensor/transducers, AtbZIP60 and AtbZIP28, are basic leucine zipper transcription factors that are activated by regulated intramembrane proteolysis systems and regulate transcription of the UPR genes. These signaling pathways play important roles not only in the UPR but also in other biological processes such as the response to pathogens and heat stress. 


\section{Introduction}

Many of membrane proteins and secretory proteins are synthesized and folded in the endoplasmic reticulum (ER). Correct folding during and after synthesis is crucial not only for production of functional proteins but also for the correct transport to target organelles. Protein folding proceeds with the assistance of molecular chaperones and other folding factors in the ER. When protein folding is inhibited because of mutations, unbalanced ratios of subunits of hetero-oligomeric proteins, disturbances in calcium homeostasis, or imbalance between the entry of nascent polypeptides and the folding capacity of the ER, unfolded proteins accumulate in the ER in an event termed "ER stress" [1,2]. Unfolded proteins are then prevented from trafficking and degraded by protein quality control systems. In addition, the overproduction of unfolded proteins is sensed by stress sensors on the ER membrane, and signal transduction pathways are activated in an attempt to maintain the homeostasis of the ER. When the ER stress is not relieved by the expression of genes that promote protein folding and removal of unfolded proteins from the ER, the apoptotic pathway is activated. Collectively, these pathways are referred to as the unfolded protein response (UPR). The molecular mechanisms underlying quality control of proteins and UPR have been described mainly in yeast and mammals. In plants, analogous pathways for quality control of proteins and UPR have been identified [3]. The quality control system and UPR in the plant ER have recently attracted considerable attention because most of the nutritional plant proteins, such as the seed storage proteins in food crops, and many therapeutic products, such as antibodies and vaccines, are synthesized in the ER. In this review, the recent 
advances in the plant ER stress signaling pathway will be summarized and discussed.

\section{Components of ER stress signaling pathways in plants}

ER stress is sensed by sensor proteins on the ER membrane. Yeast inositol-requiring enzyme-1 (IRE1) was first discovered as an ER stress sensor [4,5]. Subsequently, two additional ER stress sensors, activating transcription factor 6 (ATF6) and interferoninduced double-stranded RNA-activated protein kinase-related protein (PERK), were found to trigger UPR in mammals [6]. In plants, Ire1-like proteins and the two sensors/transducers bZIP60 and bZIP28, which are responsive to ER stress, have been identified. Plant Ire1-like proteins, Arabidopsis Ire1s (AtIre1-1 and AtIre1-2) [7,8] and rice Ire1 (OsIre1) [9], were identified based on their similarity to yeast and mammalian Ire1s. The expression of AtIre1-1 is restricted to certain tissues at specific developmental stages such as the apical meristem, the leaf margins where vascular bundles end, the anthers before pollen is formed, the ovules at an early stage of development, and the cotyledons immediately after germination. AtIre1-2 is expressed in vascular bundles of young plants, leaves, roots, seedlings, receptacles of flowers, and vascular bundles of petals. Yeast Ire1 is a transmembrane receptor protein kinase/ribonuclease located in the ER. This protein, which is activated via a process of oligomerization and autophosphorylation stimulated by ER stress, splices invalid basic leucine zipper (bZIP) transcription factor HAC1 mRNA, which is recruited by a conserved bipartite targeting element contained in the 3' untranslated region, into the mature mRNAs encoding active Hac1 [4,5,10,11]. Mammalian Ire1 also senses ER stress and splices invalid XBP1 mRNA in an analogous manner to that 
of yeast Ire1 [12]. Plant Ire1s contain the C-terminal cytosolic region, which includes the protein kinase and ribonuclease domains that are conserved from yeast to mammals. In yeast and mammals, active Hac1 or XBP1 are translated from the Ire1spliced mRNA and then translocate into the nucleus. These transcription factors bind UPR cis-activating regulatory elements in the promoter regions and induce the transcription of UPR genes $[5,13]$. The C-terminal portions of recombinant AtIre1-2 and OsIre1 exhibit autophosphorylation activity in vitro. When Lys ${ }^{442}$ of AtIre1-2 was mutated to alanine, the autophosphorylation activity was lost, suggesting that AtIre1-2 is a transphosphorylating protein kinase similar to other receptor kinases. The N-terminal domains of AtIre1-1, AtIre1-2, and OsIre1 function as ER stress sensors in yeast cells, although the amino acid sequences of the $\mathrm{N}$-terminal luminal domain of these Ire1s are not conserved between other organisms. When chimeric proteins containing the N-terminal domains of AtIre1-1, AtIre1-2, or OsIre1 and the C-terminal domain of yeast Ire1 were expressed in yeast lacking functional Ire1, treatment with tunicamycin, which results in the generation of misfolded or unfolded proteins by inhibiting $\mathrm{N}$-glycosylation, no longer inhibited growth. In addition, treatment of these cells with tunicamycin or dithiothreitol induced the UPR genes. Based on these observations, plant Ire1s are presumed to act as an ER stress sensor similar to yeast or mammalian Ire1 in vivo; however, whether plant Ire1s function as regulators of transcription during ER stress remains to be determined. Confirmation of whether AtIre1-1 functions as an ER stress sensor is challenging, because AtIRE1-1 T-DNA mutant homozygosity is lethal [14]. In contrast, an AtIRE1-1, AtIRE1-2 homozygous null mutant was obtained; however, disruption of the AtIRE1-2 gene did 
not interfere with representative ER stress-induced genes like BiP and protein disulfide isomerase (PDI) [15-17]. Unfortunately, to date, no transcription factor mRNA splicing by plant Ire1 has been found.

Mammalian ATF6 is an ER stress sensor/transducer. ATF6 is a transmembrane protein that senses ER stress through its C-terminal luminal domain and then moves to Golgi bodies to be cleaved by the serine protease site-1 protease (S1P) and the metalloprotease site-2 protease (S2P) [18] via a process termed regulated intramembrane proteolysis (RIP). The N-terminal cytosolic bZIP domain is released from the Golgi membrane into the nucleus to induce the expression of target genes [19]. In the Arabidopsis genome, 75 genes are predicted to encode bZIP transcription factors [20]. Among them, AtbZIP17, AtbZIP28, AtbZIP49, and AtbZIP60 possess a transmembrane domain (TMD). AtbZIP60 and AtbZIP28 are bZIP transcription factors that are similar to mammalian ATF6 involved in UPR, whereas AtbZIP17 is activated in response to salt stress but not ER stress [21,22]. AtbZIP49 has not been found in plants due to difficulty in isolation of cDNA [23].

AtbZIP60 protein, which is induced by ER stress, was identified as a protein by Iwata and Koizumi [24]. This 295-amino acid protein contains an N-terminal bZIP domain and a putative transmembrane domain followed by a short C-terminal luminal domain (Fig. 1). AtbZIP60 is localized as an inactive form in the ER membrane and is cleaved under ER stress [25].

Cleavage of the salt-stress sensor, AtbZIP17, by an Arabidopsis homolog of mammalian S1P following salt stress conditions was demonstrated in an in vitro pulldown assay, suggesting that a RIP-like system acts on stress signal transduction from 
the ER in plants [21]. Cleavage of AtbZIP60, however, is independent of the function of Arabidopsis homologs of mammalian S1P and S2P [25]. Cleavage of this protein was observed even in homozygous T-DNA insertion mutants of the Arabidopsis homologs of mammalian $S 1 P$ and $S 2 P$ following treatment of seedlings with tunicamycin. This finding is consistent with the lack of consensus sequence, RXXL or RXL, for S1P cleavage in AtbZIP60. Taken together, these results suggest that an unidentified processing system exists in addition to the plant S1P/S2P-dependent RIPlike system.

Another important but yet unanswered question is how AtbZIP60 senses ER stress. The ER luminal domain of ATF6 has two Golgi body localization sequences (GLS1 and GLS2). ATF6 localizes to the ER via interactions between GLS1 and BiP [26]. Thus, when unfolded proteins sequester BiP from GLS1 under ER stress, ATF6 is transported into the Golgi body and processed by RIP. On the other hand, the AtbZIP60 56-amino acid luminal domain is relatively short and lacks GLS consensus sequences. Hence, AtbZIP60 itself does not likely function as a sensor, and AtbZIP60-interacting proteins actually sense the ER stress [25]. After proteolysis, the N-terminal bZIP domain of AtbZIP60 then translocates into the nucleus. A truncated form of AtbZIP60 containing amino acids 1-216 without the transmembrane domain (AtbZIP60 $\Delta$ C) fused with green fluorescent protein (GFP) also localized to the nucleus regardless of ER stress when transiently expressed in Arabidopsis protoplasts [25]. Localization of a truncated form of the tobacco ortholog NtbZIP60 (NtbZIP60 $\Delta$ C) to the nucleus was also reported [27].

The bZIP28 protein, which is another plant ER stress sensor, is an $\mathrm{N}$ - 
glycosylated protein with a N-terminal bZIP domain, a putative TMD, and a Cterminal domain (Fig. 1) [28]. A canonical S1P cleavage site and a putative S2P cleavage site are found in the C-terminal domain and at a site adjacent to the TMD of the N-terminal domain, respectively (Fig 1). Arabidopsis bZIP28 (AtbZIP28) tagged with Myc or GFP at its N-terminus has been demonstrated to reside in the ER membrane under unstressed conditions and to be cleaved in response to ER stress induced by tunicamycin. Based on the size of the resultant AtbZIP N-terminal fragment, the plant S1P/S2P-dependent RIP system may, in fact, cleave AtbZIP28 under ER stress conditions; however, this possibility remains unproved. In addition, whether AtbZIP28 is processed in the ER or the Golgi body also remains unknown. In this system, tunicamycin and dithiothreitol treatment clearly increased GFP fluorescence in the nucleus, suggesting the translocation of the AtZIP28 N-terminal fragment to the nucleus. Furthermore, translocation of AtbZIP28 from the ER to the nucleus was indicated by the captured time-lapse imaging experiments, supporting the notion that the N-terminal fragment of AtbZIP28 translocates to the nucleus in response to ER stress [23].

Mammalian PERK is an ER transmembrane protein that senses ER stress via its luminal domain and subsequently phosphorylates a specific serine residue of translation initiation factor- $2 \alpha(\mathrm{eIF} 2 \alpha)$, resulting in general attenuation of translation [29]. Phosphorylation of eIF2 $\alpha$ also stimulates translation of ATF4 [30], which induces the transcription of many amino acid synthetic enzymes, amino acid transporters, anti-oxidation enzymes, and $C H O P$, a gene important for apoptotic cell death [31]. In plants, a similar signal transduction kinase for attenuating translation 
has not been found; however, a heterodimer of $\mathrm{G} \beta$ and $\mathrm{G} \gamma$ proteins on the ER membrane was demonstrated to be involved in the signaling events that trigger UPRassociated cell death in Arabidopsis [32]. In fact, seedlings of G $\beta$-null mutant plants are more resistant to growth inhibition by tunicamycin than wild-type plants. The connections between $\mathrm{G}$ protein signaling and the ER stress-sensing system are not understood. Despite these questions, a pathway that transduces a programmed cell death signal and diverges from the molecular chaperone-inducing branch of the UPR was postulated in soybean [33]. This integrated pathway was synergistically activated by ER stress and osmotic stresses that result in the appearance of markers associated with leaf senescence through activation of plant-specific N-rich proteins. The components of this signal transduction pathway in soybean have not yet been identified.

\section{Activation of transcription by UPR signaling}

The cis-acting regulatory element in the promoter of a UPR gene was identified first for $K A R 2$ (yeast $B i P$ ) and named UPR cis-acting regulatory element (UPRE) [3436]. Yeast UPRE is a target for HAC1. In mammalian cells, more than ten types of cis-acting regulatory elements that respond to ER stress are known [6]. Among them, UPRE (XBP1-BS), ER stress response element (ERSE), and ERSE-II are targets for both ATF6 and XBP1 [37,38]. In plants, two cis-acting regulatory elements analogous to the mammalian elements were identified. Two overlapping sequences similar to mammalian ERSE-II and UPRE was first noted in the 5' flanking sequences of the soybean BiP gene [39]. This 16-bp sequence was demonstrated to function as 
an ER stress-responsive cis-acting element and was designated plant UPR element (PUPRE) [40]. Another plant ER stress-responsive cis-acting element is ERSE, which has sequence similar to mammalian ERSE. Both P-UPRE and ERSE were found to be crucial for induction of a reporter gene in transgenic Arabidopsis treated with tunicamycin $[24,40]$.

When the truncated AtbZIP60 $\Delta \mathrm{C}$ was expressed in protoplasts of cultured tobacco cells, the promoters of the representative UPR genes, $B i P 1, B i P 2, B i P$, calnexin1 and calnexin2 were activated in a dual luciferase assay [24]. In addition, the luciferase activities driven by P-UPRE and ERSE were enhanced by the expression of AtbZIP60 $\Delta \mathrm{C}$ [24] and tobacco NtbZIP60 $\Delta \mathrm{C}$ [27]. AtbZIP60 $\Delta \mathrm{C}$ also appeared to induce the expression of AtbZIP60 through ERSE in the promoter of AtbZIP60, resulting in amplification of the signal. Expression of a truncated form of AtbZIP28 (AtbZIP28 $\Delta$ C), which contains only the cytoplasmic domain of the protein, also activated the BiP1 and BiP3 promoters via P-UPRE and ERSE in the absence of stress conditions [23]. Therefore, bZIP domains derived from both bZIP60 and bZIP28 activate the expression of the same ER stress-responsive genes.

In plants, ER stress upregulates large numbers of genes. The patterns of gene expression in normal maize and the opaque mutants $f l-2$ and $M c$, which contain mutations in the $24-\mathrm{kDa} \alpha$-zein protein or the $16-\mathrm{kDa} \gamma$-zein, were compared by DNA microarray analysis [41]. These mutations cause ER stress and result in a dramatic increase in ER-resident molecular chaperones as well as the expression of a number of stress response genes. Comprehensive analyses of the transcriptome of Arabidopsis and soybean during drug-induced ER stress revealed that genes encoding 
ER chaperones, glycosylation/modification-related proteins, translocon subunits, vesicle transport proteins, ER-associated degradation-related proteins, antiapoptosis proteins, and others were upregulated [3, 17,42-44]. Most of these genes are Arabidopsis orthologs of the yeast or mammalian UPR genes. P-UPRE and especially ERSE are found at high frequencies in the 5' flanking sequences of the genes observed to be upregulated during ER stress [3]. These analyses of the transcriptome of wild-type plants, however, may have identified genes regulated by the UPR or genes regulated by other signal transduction systems involved in crosstalk with the UPR. Therefore, transcriptome analysis of an ER stress sensor null mutant was performed to identify genes primarily regulated by the UPR. When compared with wild-type plants, homozygous bZIP60 null mutant Arabidopsis showed a markedly weaker induction of many ER-stress responsive genes [25]. Of the 129 genes activated more than three-fold by tunicamycin treatment in wild-type seedlings, 54 genes exhibited a significantly lower level of induction in the bZIP60 null mutant seedling. These affected genes encode fifteen molecular chaperones, ten protein transport-related proteins, six ER-associated degradation-related proteins, seven transcription factors, five other known proteins, and eleven unidentified proteins. While upregulation of only twelve of these genes was completely abolished by the defect in $b Z I P 60$, upregulation of the other 42 genes caused by ER stress in the bZIP60 null mutant plants were weaker than those in wild-type plants but still quite substantial, suggesting that other components responsive to ER stress, like bZIP28, simultaneously govern these genes. Indeed, Gao et al. demonstrated that overexpression of AtbZIP28 $\Delta \mathrm{C}$ in an AtbZIP28 null mutant induced ten genes 
encoding ER chaperones and other ER proteins [45]. Defects in bZIP60 exert various effects on expressions of homologous genes encoding ER molecular chaperones, which have similar functions as molecular chaperone or folding enzymes in vitro. For instance, $B i P 3 \mathrm{mRNA}$ was less strongly upregulated in the $b Z I P 60$ null mutants than in wild-type seedlings, whereas upregulation of $B i P 1$ or $B i P 2$ mRNAs was similar between the bZIP60 null mutant and the wild-type plants [25]. In addition, following ER stress, upregulation of five of the seven PDI genes was significantly attenuated in an AtbZIP60 null mutant, whereas upregulation of the other two PDI genes was unaffected [14]. The diversity in transcriptional regulation of these genes may correlate with their unique physiological roles in the ER.

In mammals, cells may be able to perform a time-dependent phase shift from the ATF6-mediated refolding phase to the XBP1-mediated refolding plus degradation phase to cope with the quality or quantity (or both) of unfolded proteins that accumulate in the ER [46-48]. Although plant cells are also expected to perform a time-dependent phase shift from one ER stress signaling pathway to the other, our lack of understanding of these pathways makes this assumption unproven at this point.

\section{Roles of the ER stress signaling pathway in biological processes other than UPR}

In mammals, the ER stress signaling pathway is involved in crosstalk with other signaling pathways and plays important roles in various biological processes. Therefore, disturbance of ER stress signaling can result in many disorders such as diabetes and neurodegenerative diseases [49]. On the other hand, examination of the 
interactions between the UPR signaling pathway and other signaling systems and the effects on biological events other than UPR is just beginning in plants. To date, crosstalk between bZIP60- or bZIP28-signaling pathway and heat-stress or pathogensignaling pathway have been identified. Thus, defective AtbZIP28 causes a striking heat-sensitive phenotype, although an AtbZIP28 null mutant was indistinguishable from wild-type plants under normal growth conditions [45]. This result suggests that AtbZIP28 contributes to heat tolerance. In plants, heat stress responses are controlled by classical heat stress transcription factors that are conserved among all eukaryotes. In addition to these transcription factors, AtbZIP28 is also upregulated under heat stress $\left(42{ }^{\circ} \mathrm{C}\right)$, and its transcription factor domain is released by the RIP-like system from the ER membrane in response to heat. As a result, expressions of genes, such as $B i P 2$, that are activated by the bZIP domain of AtbZIP28 are upregulated under heat stress. In accord with this notion, these genes were not upregulated under heat stress in the AtbZIP28 null mutant seedlings.

A tobacco or a Nicotiana benthamiana ortholog of AtbZIP60, NtbZIP60 or NbbZIP60, was identified as a component of the spermine-signaling pathway [27]. Polyamine spermine is produced and accumulates in the apoplastic space during infection with pathogens. Accumulated spermine activates two mitogen-activated protein kinases and upregulates a subset of hypersensitive response-specific genes in the spermine signaling pathway [50]. NbbZIP60 was activated by infection with a bacterial pathogen through the spermine signaling pathway. In addition, NbbZIP60silenced $N$. benthamiana plants showed decreased resistance to pathogens compared to the control plants. Hence, bZIP60 may play an important role in plant innate 
immunity. In Arabidopsis, cleavage of AtbZIP60 protein is observed in anthers even in the absence of stress treatment [25]. Hence, AtbZIP60 is also thought to function in the normal development of active secretory cells.

\section{Conclusions}

ER stress is sensed by at least two sensors/transducers, bZIP60 and bZIP28, which are located in the ER membrane in plants. In these signaling pathways, an unidentified processing system or a widely conserved RIP-like system cleaves the bZIP domain of bZIP60 or bZIP28 from the ER membrane, respectively. The released bZIP domain translocates into the nucleus and activates the UPR genes by binding to consensus sequences on their promoters. Further studies are required to elucidate the detailed molecular mechanism of these processes. The UPR pathways are also suspected to be involved in crosstalk with other transcription factors in a complex network of biological processes. Elucidation of the relationship between UPR and other biological functions will certainly offer new insight into the versatility of the plant ER.

\section{Acknowledgements}

Studies conducted in the author's laboratory were supported by a grant from the Program for Promotion of Basic Research Activities for Innovative Biosciences and a Grant-in-Aid for Exploratory Research from the Ministry of Education, Culture, Sports, Science, and Technology of Japan (18658055). 


\section{References}

[1] K. Mori (2000) Tripartite management of unfolded proteins in the endoplasmic reticulum, Cell 101, 451-454.

[2] D. Ron, P. Walter (2007) Signal integration in the endoplasmic reticulum unfolded protein response, Nat. Rev. Mol. Cell Biol. 8, 519-529.

[3] R. Urade (2007) Cellular response to unfolded proteins in the endoplasmic reticulum of plants. FEBS J. 274, 1152-1171.

[4] J. S. Cox, P. Walter (1996) A novel mechanism for regulating the activity of a transcription factor that controls the unfolded protein response, Cell 87, 391-404.

[5] K. Mori, T. Kawahara, H. Yoshida, H. Yanagi, T. Yura (1996) Signalling from the endoplasmic reticulum to the nucleus: transcription factor with a basic-leucine zipper motif is required for the unfolded protein-response pathway, Genes Cells 1, 803-817. [6] M. Schröder, R. J. Kaufman (2005) The mammalian unfolded protein response, Annu. Rev. Biochem. 74, 739-789.

[7] N. Koizumi, I. M. Martinez, Y. Kimata, K. Kohno, H. Sano, M. J. Chrispeels (2001) Molecular characterization of two Arabidopsis Ire1 homologs, endoplasmic reticulum-located transmembrane protein kinases, Plant Physiol. 127, 949-962.

[8] S. J. Noh, C. S. Kwon, W. I. Chung (2002) Characterization of two homologs of Ire1p, a kinase/endoribonuclease in yeast, in Arabidopsis thaliana, Biochim. Biophys. Acta 1575, 130-134.

[9] Y. Okushima, N. Koizumi, Y. Yamaguchi, Y. Kimata, K. Kohno, H. Sano (2002) Isolation and characterization of a putative transducer of endoplasmic reticulum stress in Oryza sativa, Plant Cell Physiol. 43, 532-539. 
[10] A. V. Korennykh, P. F. Egea, A. A. Korostelev, J. Finer-Moore, C. Zhang, K. M.

Shokat, R. M. Stroud, P. Walter (2009) The unfolded protein response signals through high-order assembly of Ire1, Nature 457, 687-693.

[11] T. Aragón, E. van Anken, D. Pincus, I. M. Serafimova, A. V. Korennykh, C. A. Rubio, P. Walter (2009) Messenger RNA targeting to endoplasmic reticulum stress signalling sites, Nature 457, 736-740.

[12] H. Yoshida, T. Matsui, A. Yamamoto, T. Okada, K. Mori (2001) XBP1 mRNA is induced by ATF6 and spliced by IRE1 in response to ER stress to produce a highly active transcription factor, Cell 107, 881-891.

[13] J. S. Cox, P. Walter (1996) A novel mechanism for regulating the activity of a transcription factor that controls the unfolded protein response, Cell 87, 391-404.

[14] D. P. Lu, D. A. Christopher (2008) Endoplasmic reticulum stress activates the expression of a sub-group of protein disulfide isomerase genes and AtbZIP60 modulates the response in Arabidopsis thaliana, Mol. Genet. Genomics 280, 199-210. [15] H. Wadahama, S. Kamauchi, M. Ishimoto, T. Kawada, R. Urade (2007) Protein disulfide isomerase family proteins involved in soybean protein biogenesis, FEBS J. 274, 687-703.

[16] H. Wadahama, S. Kamauchi, Y. Nakamoto, K. Nishizawa, M. Ishimoto, T. Kawada, R. Urade (2008) A novel plant protein disulfide isomerase family homologous to animal P5 - molecular cloning and characterization as a functional protein for folding of soybean seed-storage proteins, FEBS J. 275, 399-410.

[17] S. Kamauchi, H. Wadahama, K. Iwasaki, Y. Nakamoto, K. Nishizawa, M.

Ishimoto, T. Kawada, R. Urade (2008) Molecular cloning and characterization of two soybean protein disulfide isomerases as molecular chaperones for seed storage proteins, FEBS J. 275, 2644-2658. 
[18] J. Ye, R. B. Rawson, R. Komuro, X. Chen, U. P. Davé, R. Prywes, M. S. Brown, J. L. Goldstein (2000) ER stress induces cleavage of membrane-bound ATF6 by the same proteases that process SREBPs, Mol. Cell 6, 1355-1364.

[19] H. Yoshida, T. Okada, K. Haze, H. Yanagi, T. Yura, M. Negishi, K. Mori (2000) ATF6 activated by proteolysis binds in the presence of NF-Y [CBF] directly to the cisacting element responsible for the mammalian unfolded protein response, Mol. Cell Biol. 20, 6755-6767.

[20] M. Jakoby, B. Weisshaar, W. Dröge-Laser, J. Vicente-Carbajosa, J. Tiedemann, T. Kroj, F. Parcy (2002) bZIP transcription factors in Arabidopsis, Trends Plant Sci. 7, 106-111.

[21] J. X. Liu, R. Srivastava, P. Che, S. H. Howell (2007) Salt stress responses in Arabidopsis utilize a signal transduction pathway related to endoplasmic reticulum stress signaling, Plant J. 51, 897-909.

[22] J. X. Liu, R, Srivastava, S. H. Howell (2008) Stress-induced expression of an activated form of AtbZIP17 provides protection from salt stress in Arabidopsis, Plant Cell Environ. 31, 1735-1743.

[23] H. Tajima, Y. Iwata, M. Iwano, S. Takayama, N. Koizumi (2008) Identification of an Arabidopsis transmembrane bZIP transcription factor involved in the endoplasmic reticulum stress response, Biochem. Biophys. Res. Commun. 374, 242-247.

[24] Y. Iwata, N. Koizumi (2005) An Arabidopsis transcription factor, AtbZIP60, regulates the endoplasmic reticulum stress response in a manner unique to plants, Proc. Natl. Acad. Sci. U S A 102, 5280-5285. 
[25] Y. Iwata, N. V. Fedoroff, N. Koizumi (2008) Arabidopsis bZIP60 is a proteolysisactivated transcription factor involved in the endoplasmic reticulum stress response, Plant Cell 20, 3107-3121.

[26] J. Shen, X. Chen, L. Hendershot, R. Prywes (2002) ER stress regulation of ATF6 localization by dissociation of BiP/GRP78 binding and unmasking of Golgi localization signals. Dev. Cell 3, 99-111.

[27] C. Tateda, R. Ozaki, Y. Onodera, Y. Takahashi, K. Yamaguchi, T. Berberich, N. Koizumi, T. Kusano (2008) NtbZIP60, an endoplasmic reticulum-localized transcription factor, plays a role in the defense response against bacterial pathogens in Nicotiana tabacum, J. Plant Res.121, 603-611.

[28] J. X. Liu, R. Srivastava, P. Che, S. H. Howell (2007) An endoplasmic reticulum stress response in Arabidopsis is mediated by proteolytic processing and nuclear relocation of a membrane-associated transcription factor, bZIP28, Plant Cell 19, 41114119.

[29] H. P. Harding, Y. Zhang, A. Bertolotti, H. Zeng, D. Ron (2000) Perk is essential for translational regulation and cell survival during the unfolded protein response, Mol. Cell 5, 897-904.

[30] H. P. Harding, Y. Zhang, H. Zeng, I. Novoa, P. D. Lu, M. Calfon, N. Sadri, C. Yun, B. Popko, R. Paules, D. F. Stojdl, J. C. Bell, T. Hettmann, J. M. Leiden, D. Ron (2003) An integrated stress response regulates amino acid metabolism and resistance to oxidative stress, Mol. Cell 11, 619-633. 
[31] Y. Ma, J. W. Brewer, J. A. Diehl, L. M. Hendershot (2000) Two distinct stress signaling pathways converge upon the $\mathrm{CHOP}$ promoter during the mammalian unfolded protein response, J. Mol. Biol. 318, 1351-1365.

[32] S. Wang, S. Narendra, N. Fedoroff (2007) Heterotrimeric G protein signaling in the Arabidopsis unfolded protein response, Proc. Natl. Acad. Sci. U S A 104, 38173822

[33] M. D. Costa, P. A. Reis, M. A. Valente, A. S. Irsigler, C. M. Carvalho, M. E. Loureiro, F. J. Aragão, R. S. Boston, L. G. Fietto, E. P. Fontes (2008) A new branch of endoplasmic reticulum stress signaling and the osmotic signal converge on plantspecific asparagine-rich proteins to promote cell death, J. Biol. Chem. 283, 2020920219.

[34] K, Kohno, K. Normington, J. Sambrook, M.-L. Gething, K. Mori K (1993) The promoter region of the yeast KAR2 (BiP) gene contains a regulatory domain that responds to the presence of unfolded proteins in the endoplasmic reticulum, Mol. Cell Biol. 13, 877-890.

[35] K. Mori, N. Ogawa, T. Kawahara, H. Yanagi, T. Yura (1998) Palindrome with a spacer of one nucleotide is characteristic of the cis-acting unfolded protein response element in Saccharomyces cerevisiae, J. Biol. Chem. 273, 9912-9920.

[36] K. Mori, A. Sant, K. Kohno, K. Normington, M. J. Gething, J. F. Sambrook (1992) A 22 bp cis-acting element is necessary and sufficient for the induction of the yeast KAR2 (BiP) gene by unfolded proteins, EMBO J. 11, 2583-2593.

[37] H. Yoshida, K. Haze, H. Yanagi, T. Yura, K. Mori (1998) Identification of the cisacting endoplasmic reticulum stress response element responsible for transcriptional 
induction of mammalian glucose-regulated proteins: Involvement of basic leucine zipper transcription factors, J. Biol. Chem. 273, 33741-33749.

[38] K. Yamamoto, H. Yoshida, K. Kokame, R. J. Kaufman, K. Mori (2004)

Differential contributions of ATF6 and XBP1 to the activation of endoplasmic reticulum stress-responsive cis-acting elements ERSE, UPRE and ERSE-II, J. Biochem. 136, 343-350.

[39] R. A. A. Buzeli, J. C. M. Cascardo, L. A. Z. Rodrigues, M. O. Andrade, R. S. Almeida, M. E. Loureiro, W. C. Otoni, E. P. B. Fontes (2002) Tissue-specific regulation of $\mathrm{BiP}$ genes: a cis-acting regulatory domain is required for $\mathrm{BiP}$ promoter activity in plant meristems, Plant Mol. Biol. 50, 757-771.

[40] D. H. Oh, C. S. Kwon, H. Sano, W. I. Chung, N. Koizumi (2003) Conservation between animals and plants of the cis-acting element involved in the unfolded protein response, Biochem. Biophys. Res. Commun. 301, 225-230.

[41] B. G. Hunter, M. K. Beatty, G. W. Singletary, B. R. Hamaker, B. P. Dilkes, B. A. Larkins, R. Jung (2002) Maize opaque endosperm mutations create extensive changes in patterns of gene expression, Plant Cell 14, 2591-2612.

[42] I. M. Martìnez, M. J. Chrispeels (2003) Genomic analysis of the unfolded protein response in Arabidopsis shows its connection to important cellular processes, Plant Cell 15, 561-576.

[43] S. Kamauchi, H. Nakatani, C. Nakano, R. Urade R (2005) Gene expression in response to endoplasmic reticulum stress in Arabidopsis thaliana, FEBS J. 272, 34613476. 
[44] A. S. Irsigler, M. D. Costa, P. Zhang, P. A. Reis, R. E. Dewey, R. S. Boston, E. P. Fontes (2007) Expression profiling on soybean leaves reveals integration of ER- and osmotic-stress pathways, BMC Genomics 8, 431.

[45] H. Gao, F. Brandizzi, C. Benning, R. M. Larkin (2008) A membrane-tethered transcription factor defines a branch of the heat stress response in Arabidopsis thaliana, Proc. Natl. Acad. Sci. U S A105, 16398-16403.

[46] K. Yamamoto, T. Sato, T. Matsui, M. Sato, T. Okada, H. Yoshida, A. Harada, K. Mori (2007) Transcriptional induction of mammalian ER quality control proteins is mediated by single or combined action of ATF6alpha and XBP1, Dev. Cell 13, 365376.

[47] A. H. Lee, N. N. Iwakoshi, L. H. Glimcher (2003) XBP-1 regulates a subset of endoplasmic reticulum resident chaperone genes in the unfolded protein response, Mol. Cell Biol. 23, 7448-7459.

[48] H. Yoshida, T. Matsui, N. Hosokawa, R. J. Kaufman, K. Nagata, K. A. Mori (2003) A time-dependent phase shift in the mammalian unfolded protein response, Dev. Cell 4, 265-271.

[49] H. Yoshida (2007) ER stress and diseases, FEBS J. 274, 630-658.

[50] Y. Takahashi, T. Berberich, A. Miyazaki, S. Seo, Y. Ohashi, T. Kusano (2003) Spermine signalling in tobacco: activation of mitogen-activated protein kinases by spermine is mediated through mitochondrial dysfunction, Plant J. 36, 820-829. 


\section{Figure legends}

Fig. 1. Diagrams of the structures of Arabidopsis ER stress sensors. AtIRE1-1 and AtIRE1-2 are type I transmembrane proteins. AtbZIP60 and AtbZIP28 are type II transmembrane proteins. AtbZIP28 contains a canonical S1P and a putative S2P site (indicated by arrows). TMD indicates the conserved transmembrane domain, and bZIP indicates the basic leucine zipper motif.

Fig. 2. Model for the ER stress-induced activation of bZIP60 and bZIP28. The bZIP60 and bZIP28 proteins localize to the ER membrane. Following accumulation of unfolded proteins in the ER, the N-terminal cytoplasmic domains of bZIP28 and bZIP60 are released from the ER membrane by a S1P/S2P-dependent RIP-like mechanism or another unknown mechanism. Transportation of bZIP28 to the Golgi body for cleavage by S1P has not yet been confirmed. Both N-terminal cytoplasmic domains released from bZIP60 and bZIP28 are translocated to the nucleus and induce transcription of UPR genes that possess P-UPRE and ERSE regulatory elements in the promoter. Transcription of bZIP60 and bZIP28 is activated by infection with pathogens and heat stress, respectively. Hence, transcription of the same UPR genes is activated as a result of ER stress, heat stress, and infection of pathogens. 
Fig. 1. Diagrams of the structures of Arabidopsis ER stress sensors. AtIRE1-1 and AtIRE1-2 are type I transmembrane proteins. AtbZIP60 and AtbZIP28 are type II transmembrane proteins. AtbZIP28 contains a canonical S1P and a putative S2P site (indicated by arrows). TMD indicates the conserved transmembrane domain, and bZIP indicates the basic leucine zipper motif. $155 \times 110 \mathrm{~mm}(200 \times 200$ DPI $)$ 
Fig. 2. Model for the ER stress-induced activation of bZIP60 and bZIP28. The bZIP60 and bZIP28 proteins localize to the ER membrane. Following accumulation of unfolded proteins in the ER, the $\mathrm{N}$-terminal cytoplasmic domains of bZIP28 and bZIP60 are released from the ER membrane by a S1P/S2P-dependent RIP-like mechanism or another unknown mechanism. Transportation of bZIP28 to the Golgi body for cleavage by S1P has not yet been confirmed. Both N-terminal cytoplasmic domains released from bZIP60 and bZIP28 are translocated to the nucleus and induce transcription of UPR genes that possess P-UPRE and ERSE regulatory elements in the promoter. Transcription of bZIP60 and bZIP28 is activated by infection with pathogens and heat stress, respectively. Hence, transcription of the same UPR genes is activated as a result of ER stress, heat stress, and infection of pathogens.

$120 \times 152 \mathrm{~mm}(200 \times 200 \mathrm{DPI})$ 


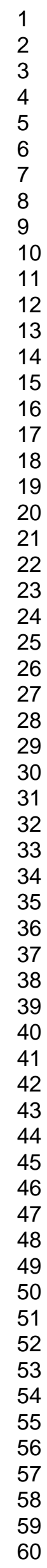

